\title{
Energy, causality and the living cell: the Schrödinger Machine concept
}

\author{
Alexander Powell \\ ap.cybercraft@googlemail.com \\ ORCiD: https://orcid.org/0000-0001-6911-1890 \\ 10 March 2018 \\ (Version 1: 27 February 2018)
}

\begin{abstract}
The ability to stave off, if only for a time, the seemingly inevitable eventual decay to thermodynamic equilibrium is one of the chief distinguishing characteristics of life. That it is achieved in large part through the exploitation of ambient thermal energy is generally under-appreciated, however. The ATP that powers the cell, meanwhile, is still often described as the bearer of high-energy bonds, when even if that were true it is in any case a quite different factor that underlies its motive capacity. This article aims to act as a corrective with respect to both of those points, whilst also providing a broad-brush perspective on causality in the living cell. It is proposed that the living cell may be regarded as the exemplification of a particularly distinctive kind of machine, here termed Schrödinger Machine.
\end{abstract}

Keywords: life, thermodynamics, metabolism, ATP, bioenergetics, molecular dynamics, biocausality, thermo-catalytic ratchet, Schrödinger Machine, synthetic biology

\section{Introduction}

Perhaps the most fundamental question in biology is that posed by the title of Schrödinger's famous monograph: 'What is life?' (Schrödinger 1944). Today, thanks to the combined efforts of generations of cell biologists, biochemists, molecular geneticists, biophysicists, physiologists, evolutionary biologists, and other bioscience researchers, it often feels as though we more or less know the answer. At any rate, we know pretty well what living organisms are made of down to the molecular level, and our knowledge of how the matter of life is organized is rapidly being fleshed out. Undoubtedly there are gaps, and still we occasionally learn of the existence of entire new classes of biomolecules, or gain fresh insight into the functional significance of, or relationships between, those cell entities we already know about. But on the whole there seem to be few aspects of cell function that we cannot associate with known entities or classes of entities. Developments such as the 'resolution revolution' in 
cryo-electron microscopy (Kühlbrandt 2014; Brzezinski 2017) make the prospects for obtaining detailed structures for all biomolecules better than ever. Rather more abstractly, it seems plausible to point to metabolism, and to an organism's place within a lineage defined in terms of genomic relationships, as together being essentially criterial of life itself (O'Malley \& Dupré 2009).

Casting a shadow over this heart-warming epistemic scene, however, is the fact that many important questions remain rather hard to answer satisfactorily. For a start, how does development take the course it does? Or then again, what is disease, and what is a pathogen? (Scully 2004; Méthot \& Alizon 2014) And more basic even than those: why, without exception, do we die? Relevant to that last question is the fact that when we die, our bodies attain thermodynamic equilibrium with their surroundings. So, as Schrödinger nicely articulated, what the living organism fundamentally succeeds in doing, in its vitality, is stave off the decay to thermodynamic equilibrium (Schrödinger 1944, p.69). All the capacities and characteristics of living organisms that are salient to their survival depend ultimately on the maintenance of a state of dis-equilibrium with their environment. We die when and because we lose the capacity to maintain such a state. That idea is somewhat abstract, however, as is Schrödinger's notion that the organism maintains its non-equilibrium state by feeding on 'negative entropy' in the form of food molecules (ibid, p.70). What do such formulations mean in concrete terms, and how might they be elaborated to impart a real sense of understanding?

\section{The cell as a self-renewing entity}

Let's begin by recapitulating some of what we know about the eukaryotic cell (for example an amoeba). ${ }^{1}$ It is more than just a bag of enzymes, but its boundary with the environment is nonetheless defined by the plasma membrane, a phospholipid bilayer which serves to contain the fluid cytoplasm. The cytoplasm contains a variety of functionally specialized membranebounded organelles, including the nucleus containing the genome, the mitochondria where energy is generated, and other functional compartments in which cell components are assembled, processed or broken down. A proteinous cytoskeleton imparts a degree of dynamic structuring on the cytoplasmic space, and is involved in the movement of various components around the cell. The cell constantly takes up specific molecules from the environment and transforms them into new molecular species. Some of these are released into the environment as waste products or, depending on the cell type, as functionally important outputs, e.g. signalling molecules or digestive enzymes. The molecules that are retained perform important structural or functional roles in the cell, and confer specific behavioural and adaptive capacities on it. Sometimes cell structures self-assemble, while the construction of others is associated with a net energy cost. The complex network of interlinked reactions that take place within the cell, and which indeed to a significant extent are constitutive of it, is what we term metabolism. As is well known, metabolic reactions are usually catalysed by enzymes, which are proteins whose amino acid sequences are encoded in the

\footnotetext{
${ }^{1}$ Although I focus at various places, as here, on the eukaryotic cell, my general perspective on cell causality - in particular the points I make regarding ATP's mode of action and the way cells harness thermal energy - applies no less to prokaryotes.
} 
genome. ${ }^{2}$ Different sets of proteins are expressed by different cells in different conditions, and much of the DNA making up the genome is involved in the regulation of gene expression.

Such a summary as the above is a gross simplification, of course, and necessarily so. But even in its cartoonishness it contains important points to ponder. For one thing it carries more than a hint that the cell is a dynamic entity, being largely fluid and composed of continuous processes involving molecular synthesis, movement, transformation and degradation. Nonetheless we might come away with the sense that while the cell's contents change, at least its membranous border is relatively well-defined and stable. That would be wrong, however: even the cell membrane is constantly turned over and renewed. In animal cells roughly half of the total membrane phospholipid is replaced every few cell divisions (Dawidowicz 1987). It has been reported that in plant cells complete turnover of the plasma membrane occurs over time scales ranging from just minutes to hours (Steer 1988). Lipid vesicles continuously pinch off from the membrane to be internalized for processing and degradation, and in the reverse direction vesicles flow continuously outwards to fuse with the membrane. The picture we obtain is one in which the cell perpetuates itself by an ongoing process of renewal that involves the continuous turnover and throughput of matter.

It is tempting to suppose that at least the genome is a stable entity, and in general it is true to say that the DNA sequence itself is impressively invariant - because sequence changes would be liable to either disrupt the structures of expressed proteins or interfere with the logic of gene expression. But this invariance is hard-won, reflecting the action of a battery of ongoing maintenance operations that combat chance mutations, copy errors, transposition events, and other sequence disruptors. Moreover, some genomic components are highly dynamic. For example, the acetyl groups on histones at gene promoters have a half-life of the order of minutes thanks to the action of competing enzymes that catalyse their addition and removal (Natoli 2010). The upshot is that under close analysis the genome, in its cellular context, looks less like a stable information repository and more like a rewritable data storage device (Shapiro 2011).

In the process of renewing itself through the continuous turnover of its constituent entities the cell preserves certain aspects of itself, even as it changes in response to the nature of the sensed environment. Preservation of genome sequence was noted above, and it is largely because of this that the cell is able to preserve its biological capacities. Proximately, protein levels reflect a balance between continuous and competing processes of synthesis and degradation. ${ }^{3}$ Thus the functional competence of the standing complement of proteins and other molecules that make up the cell is maintained, at least up to a point, in the face of chemical wear and tear, for example from reactive oxygen derivatives (Murphy 2009). But in addition what is preserved is the evolved capacity of the organism to renew itself in an adaptive way by selecting from its repertoire of gene expression patterns that pattern most compatible

\footnotetext{
${ }^{2}$ I gloss over the various possibilities for post-transcriptional and post-translational processing which may occur.

${ }^{3}$ This may be done not just to ensure that the extant stock of protein is 'fresh' and functional, but also in order to permit more rapid and effective regulation of levels (Hinkson \& Elias 2011).
} 
with its current situation, whatever that happens to be, with a fidelity that accurately reflects the vicissitudes of the organism's evolutionary past. ${ }^{4}$ This depends on the logic of gene expression and regulation, and its coupling to cell signalling systems. In metazoans, complex gene regulatory network define distinct cell phenotypes that represent attractors in a highdimensional state space (Huang et al. 2005). One genome can encode a multitude of cell identities or states, qua sets of expressed genes, and this constitutes one way in which the cell is able to adapt (within limits) to the nature of its environment. In addition, superimposed on this, is the second-order tuning of cell state, especially highly developed in eukaryotic cells, that may be achieved by regulating biomolecular properties via alternations to their phosphorylation states. These are effected by a diverse array of kinases, phosphatases and phosphorylases.

The relationship between the turnover of cell constituents and the processes of cell growth and reproduction is an intriguing area for speculation. In bacteria, growth and cell division appear to be coupled quite closely, although the link with material turnover is perhaps less clear. It has been found, however, that $E$. coli can replace its outer membrane proteins (OMPs) in just two cell generations, and OMP turnover appears to be coupled to growth and division. The mechanism involves old OMPs being displaced to the poles of growing cells, with new OMPs being inserted into the central region of the cell (Rassam et al. 2015). ${ }^{5}$ In metazoans, on the other hand, perhaps material turnover is more decoupled from cell growth. Schrödinger noted that one of the characteristic features of matter we deem to be living is that it "goes on 'doing something', moving, exchanging material with its environment, and so forth ... for a much longer period than we would expect an inanimate piece of matter to 'keep going' under similar circumstances" (Schrodinger 1944, p.69). To speculate somewhat, perhaps cell growth, cell division and the material turnover of cell constituents can be seen as representing different ways of fulfilling a requirement for non-equilibrium processes to simply carry on. Where cell growth or division are somehow constrained, for example in metazoans because of a requirement that cells be good neighbours, perhaps turnover takes on a greater importance as what could be termed a dynamics outlet. ${ }^{6,7}$

\footnotetext{
${ }^{4}$ Perhaps, and here I conjecture, the cell has to implement some kind of trade-off between selecting the state that is optimal for its current situation and the state that gives it most room for manoeuvre in terms of the range of future states accessible from that state. (This is a general point that applies to multicellular organisms as much as single cells. If your environment is liable to change, you had better be able to adapt quickly and well.)

5 See also commentary at https://phys.org/news/2015-06-bacterial-outer-membrane-proteins.html (last accessed 23/02/2018).

${ }^{6}$ Speculating still further, perhaps it would pay us to widen our optic and consider a lineage of directly related organisms as constituting a single ongoing material process, spatiotemporally chunked into what we perceive to be individuals within the lineage but in fact connected in molecular process terms. Then it may become interesting to think about renewal more broadly. Does it make sense to regard each lineage member as a necessary re-expression of the genome(s) of its parent(s) that, in renewal terms, in some way stands comparison with the way in which an individual lineage member's constituent molecules are refreshed through the re-expression of genes?

${ }^{7}$ Where warm-blooded organisms are concerned, there is presumably a functional link here with thermoregulation.
} 


\title{
ATP as a metabolic driver
}

The crude thumbnail sketch of eukaryotic cell physiology given earlier mentioned the generation of energy by the mitochondria. Regarding that topic, it is widely understood that metabolism is in some sense powered by ATP. In eukaryotes the bulk of the ATP is synthesized by the mitochondrial F-ATPase using the energy provided by the proton gradient established across the mitochondrial inner membrane by oxidative phosphorylation. ${ }^{8}$ The basic idea, when it is claimed that ATP powers metabolism, is that some reaction that would otherwise be thermodynamically unfavourable is rendered favourable if it coupled to the hydrolysis of ATP to ADP and Pi. Certainly it is the case that the hydrolysis of ATP is associated with a net negative change in free energy (let us call this quantity $\mathrm{X}$ ), which can in principle be traded against a net positive change in free energy for a coupled reaction (let us call it $Y$ ) in order to yield an overall negative free energy change (provided $|X|>|Y|$ ), i.e. ATP can indeed make for thermodynamic favourability.

Sometimes, however, it is implied or stated, even in seemingly authoritative places, ${ }^{9}$ that the bond between ADP and Pi is 'high energy'. It is suggested that it is the energy liberated by cleavage of this supposed high-energy bond that powers the particular reaction in question. The misunderstanding is widespread and has been noted by others (Storey 1992; Cooper \& Klymkowsky 2013; Dreyfus et al. 2014). Yet as bioenergeticist David Nicholls made clear some years ago, this picture is erroneous:

\begin{abstract}
It is frequently, and misleadingly, supposed that the phosphate anhydride bonds of ATP are "high-energy" bonds which are capable of storing energy and driving reactions in otherwise unfavourable directions. However, it should be clear from Table 3.1 that it is the extent to which the observed mass-action ratio is displaced from equilibrium which defines the capacity of the reactants to do work, rather than any attribute of a single component. ...
\end{abstract}

(Nicholls, 1982, p.48)

So the important point about ATP as a fuel source or energy currency in the cell is not that it contains especially distinctive bonds. (It does not.) Rather, in addition to the fact that ATP hydrolysis is associated with a net negative free energy change, which typically is all to the good, there is the mass-action ratio effect mentioned by Nicholls. In that regard we note that if ATP and ADP+Pi were in equilibrium, they would exist in a ratio of 10 million to 1 of ADP to ATP (Nicholls \& Ferguson 2013, p.33). What is crucial to ATP's physiological role is that cells

\footnotetext{
${ }^{8}$ ATP export from the mitochondria is coupled to ADP import via the ADP/ATP translocases that make up around $10 \%$ of the protein in the inner mitochondrial membrane. (The membrane is otherwise impermeable to ATP and ADP.)

${ }^{9}$ See, for example:

https://chem.libretexts.org/Core/Biological_Chemistry/Metabolism/Important_High_Energy_Molecules in Metabolism (cached at http://www.webcitation.org/6xlgsYRij on 8 March 2018);

https://chem.libretexts.org/Core/Biological_Chemistry/Metabolism/ATP\%2F\%2FADP (cached at http://www.webcitation.org/6xlglrkwk on 8 March 2018);

http://academic.brooklyn.cuny.edu/biology/bio4fv/page/atp.htm (cached at http://www.webcitation.org/6xlgftFD1 on 8 March 2018).
} 
are so effective in converting ADP to ATP, to the extent that in the eukaryotic cell the cytoplasmic ratio of ADP to ATP becomes something like 1 to 1000 (ibid, p.33). Rough calculations suggest that in order to do this the mitochondria in each of the approximately 12 trillion cells of the human body that are not red blood cells (which lack mitochondria) must synthesize tens of millions of ATP molecules per second. ${ }^{10}$ As a result, a typical adult synthesizes more than their own body weight in ATP every day! (Törnroth-Horsefield and Neutze 2008)

Now, enzymes catalyse reactions in both the forwards and backwards directions ${ }^{11}$; they merely lower the energy barrier to interconversion, in part by stabilizing the transition state (Pauling 1946, p.1377). If an enzyme-catalysed reaction is coupled in its (physiologically) forwards direction with ATP hydrolysis, then when ATP is preponderant over ADP this will heavily bias events in favour of that forwards reaction. The excess of ATP over ADP in the cytoplasm thus means that ATP acts as a driver for all the metabolic reactions coupled to its hydrolysis. If, in contrast, ATP and ADP were in equilibrium then the vast excess of ADP over ATP would drive very few metabolic pigs to market! The implication of this mode of action of ATP in the cell is that, as Nicholls noted, any molecule could in principle have been used by the cell as the energy currency (Nicholls 1982, p.48). It may just be a matter of evolutionary contingency that ATP was selected.

\section{Thermal energy and molecular dynamics}

If ATP is one misunderstood causal player within the cell, then I suggest that thermal energy is another. At temperatures above absolute zero, thermal energy manifests as molecular motion. Indeed temperature just is a measure of the amount of motion. Thus the molecules of the cell are continually moving about, rotating and vibrating, according to the physico-chemical constraints on their mobility implied by the dynamic composition of the cytoplasmic space and, in particular, the phenomenon of molecular crowding (Zhou et al. 2008). It would be naïve to conceive of the cytoplasm in terms of the bulk properties of simple liquids, but nevertheless it makes sense to imagine larger macromolecules drifting and tumbling about in the fluid cytoplasm, their domains moving relative to one another, while smaller molecules and ions zoom around much faster. Molecules collide and deflect off one another, so that over time, and over short distances, they follow the random trajectories described as Brownian motion (Di Rienzo et al. 2014). Single chemical bonds constitute axes around which chemical groups, or larger parts of molecules, spin around. Parts of molecules of various scales oscillate, with

\footnotetext{
${ }^{10}$ It has been estimated that the average human body contains around 37trn cells of all types (Bianconi et al. 2013). A typical human body contains around 5 litres of blood, each microlitre of which contains around $5 \mathrm{~m}$ red blood cells (RBCs) (https://www.nhs.uk/conditions/red-blood-count/ [last accessed 8 March 2018]). Therefore the typical human contains around 25trn RBCs, implying a total non-RBC cell count for the human body of around 12 trn. Now if $9 \times 10^{20}$ ATP molecules are synthesized per second (whole body) (Rich 2003) then each cell that is not an RBC synthesizes around 75m ATP molecules per second on average.

${ }^{11}$ For example, many of the enzymes involved in glycolytic reactions are also capable of doing duty in gluconeogenesis, where they catalyse the glycolytic reactions in reverse.
} 
smaller or more tightly bound groups vibrating with higher frequencies than more massive or less tightly bound groups (McCammon \& Harvey 1987).

Schrödinger wondered how living systems withstand these dynamic effects of thermal energy and the 'spontaneously' favourable chemical reactions they allow to take place. He suspected that the solid state plays a role in ensuring the preservation of order, in virtue of the strong chemical bonds that maintain that state in the face of ambient thermal energy. However, it is important to recognise that far from having just to withstand thermal energy - and of course beyond certain limits attempt to withstand it they must - living systems do in fact, within those limits, harness it to further their own ends. Indeed, without thermal energy and the multi-scale molecular dynamics with which it is associated, cell events and processes would grind to a halt. Consider, for example, protein folding, the process whereby many polypeptides, under physiological conditions, converge on a single three-dimensional folded structure termed the native conformation (Dobson 2003). To do so requires journeying through conformational space (CS), a vast, virtual, multi-dimensional space, the dimensions of which are defined by a polypeptide's different possible bond angles and lengths. Some conformations - points in CS - are more stable (have a lower potential energy) than others, for example because they satisfy the hydrogen-bonding potential of more amino acid residues or because they involve the more complete burial of hydrophobic residues than do other conformations. The native conformation is commonly considered the minimum energy conformation under physiological conditions.

In the case of some very fast folding polypeptides, folding is always 'downhill' in potential energy terms - there are few or no major potential energy maxima or minima to be traversed in CS (Muñoz \& Cerminara 2016). For many polypeptides, however, reaching the native conformation from other conformations requires the traversal or negotiation of perhaps substantial potential energy peaks and troughs. (For example, a non-native conformation might nonetheless be stabilised by a large number of hydrogen bonds, and to break free from this conformation to attain the native conformation requires an input of energy.) This is where thermal energy comes in. For without the structural dynamics that any molecular system exhibits at temperatures above absolute zero (let us call this thermal energy-associated molecular dynamics, or TE-MD for short), unfolded polypeptides would frequently become stuck in local energy minima. It is thermal jiggling and wiggling, to quote Richard Feynmann ${ }^{12}$, that enables a polypeptide to attain the native conformation.

TE-MD not only enables proteins to fold, it is also essential for many molecular recognition processes. Here the story is in some sense the complement of the above account of protein folding, for increasingly it appears that the molecular recognition involved in cell signalling systems, for example, depends on polypeptides which do not fold into a single welldefined conformation under physiological conditions (Wright \& Dyson 2015). These so-called intrinsically disordered proteins (IDPs) sample a wide range of conformations under such conditions, a characteristic that stems from the presence of a high proportion of flexibility-

12 "[E]verything that living things do can be understood in terms of the jigglings and wigglings of atoms" (Feynmann Lectures in Physics, Chapter 3). 
promoting amino acids such as proline, lysine and serine (Linding et al. 2003). In addition many proteins contain flexible loop regions between regions of well-defined secondary structure.

The best way to characterize molecular recognition involving IDPs and loop regions, I suggest, is as an exploratory process that depends on, indeed is driven by, molecular dynamics. And that, in turn, depends on thermal energy. Weak initial binding is followed by conformational reconfiguration of the interacting molecules and a progressive increase in binding area as the process proceeds. This is not so much molecular docking as molecular surface melting. Without TE-MD, the sampling of macromolecular conformational space involved in recognition and binding processes would simply not occur. There is a connection here with enzyme catalysis, in that it too involves molecular recognition and binding events, between enzyme and substrate(s). And again, the contemporary picture is that enzymesubstrate interactions involve sometimes substantial structural modification of one or both interaction partners. Fischer's metaphor of lock and key is clearly too rigid to be descriptive of such phenomena, whereas Koshland's induced fit model seems far more felicitous (Koshland 1995).

As for enzyme catalysis itself, the jury is arguably still out on the question of whether dynamics plays a decisive role in the ability of enzymes to achieve the extraordinary levels of rate enhancement sometimes seen. ${ }^{13}$ However, empirical findings have been interpreted by some as evidence of the importance of dynamics as a facilitator of quantum tunnelling in certain classes of enzyme-catalysed reaction (Hay \& Scrutton 2012; Klinman \& Kohen 2013; Klinman et al. 2017). Some have questioned such interpretations (Glowacki et al. 2012; Warschel \& Bora 2016), while others have argued ecumenically that while some enzymes are likely to depend on dynamics to achieve efficient catalysis, others, such as those in which catalysis involves the establishment of an electrostatic field that is brought to bear on the substrate(s), are likely to exhibit comparative stability. An interesting case is dihydrofolate reductase, which is able to cycle through various different conformations in order to target and position different substrates. The hydride transfer reaction that it catalyses, however, takes place in the context of a specific conformation in which a sizable electric field is exerted (Fried \& Boxer 2017). What we can say is that enzymes are dynamic entities subject to motions occurring over a wide range of timescales, from seconds to femtoseconds, and that such motions can play a key role at various stages in the catalytic process, including substrate binding, structural rearrangement towards the transition state, product release, etc. (Kohen 2014; Callender \& Dyer 2015; Zhao 2017). The conformational sampling to which TE-MD gives rise looks increasingly to be, in various ways, an ineluctable causal component of catalysis in the living cell.

\section{A model of cell causality}

The two causal actors just discussed - ATP as metabolic driver and TE-MD as mechanism for exploring the structural state space of the cell - are of sufficient importance to take centre stage in a model of cell causality that if not wholly novel has to date nonetheless gone virtually unstated. One might colloquially term it the 'jiggle/react' model, or it could be spoken of more

${ }^{13}$ Rate enhancements of up to $10^{26}$ have been observed (Klinman et al. 2017). 
formally as the thermo-catalytic ratchet model. Both phrases highlight what is going on in the living cell, although the ratchet concept has a particular resonance. ${ }^{14}$ Now a ratchet is a device that permits some kind of forward motion by a mechanism (e.g. a gear wheel) but resists the mechanism's reversal, and this resistance to reversal is precisely what is required of cell metabolism if dis-equilibrium is to be maintained and equilibrium, i.e. death, avoided.

When metabolic reactions run in a physiologically desirable direction the cell's reaction networks are coupled in a mutually supportive fashion to realise what we might (in a teleological frame of mind) describe as the ultimate functional objectives of the cell, i.e. continued adaptive existence, through the means of sustained molecular flux. Glucose and oxygen are consumed, and carbon dioxide and water produced (assuming aerobic respiration); gene expression occurs, proteins are produced, and molecular processing takes place as it should. We have seen how ATP delivers the motive chemical force that powers this great flux. Each metabolic reaction that occurs represents a click of the ratchet of life, with reactants becoming products and the cell advancing to a new state. But, as noted in the previous section, for reactants to encounter each other and combine propitiously, for products to disperse and reach their destinations, for interactants to reconfigure in apt ways, requires molecular dynamics driven by thermal energy. So we can see that it is Feynmann's 'jigglings and wigglings' that enable the cell to get to a state where a particular metabolic reaction can occur; and when it occurs, the cell state advances by an increment. The cytoplasmic excess of ATP to ADP, achieved ultimately in both prokaryotes and eukaryotes by harnessing the energy released by membrane-localized redox events to create a trans-membrane proton gradient, helps to prevent backsliding. Dis-equilibrium gains are locked in so that further jigglings and wigglings will, in aggregate at least, take the cell to other dis-equilibrium states, not backwards to equilibrium, and reaction partners can proceed on to the next stage of their metabolic journeys.

Thus we may plausibly conjecture that the organism, at the level of the cell, transduces thermal energy in order to realise, in conjunction with ATP, the reactions that maintain the molecular flux, and thereby avoid equilibrium. There are other significant factors, of course. Critically important is the topology of the metabolic network. That it has a circular character has been dwelt on by others (e.g. Athel Cornish-Bowden and co-workers), and this is connected with the obvious sense in which the cell, and the organism, largely retains its overall molecular type-level identity - to overlook, where metazoans are concerned, the processes of development and aging that overlay this, and to overlook the type-level changes that occur in the cell as it adapts its state to its surroundings - even as the underlying molecular tokens are continuously brought into and removed from service. Metabolic reactions are vectors that take biomolecules into the future; they ensure that a molecule's present structure is not an endpoint but merely a stage on a journey. And yet the overall architecture of metabolic circularity means that after catabolism of food molecules, and anabolism of cell structures, comes

\footnotetext{
14 The suggestive phrase 'Brownian ratchet' is already in use, and has historically had - if the Wikipedia entry for the term [last accessed 24 February 2018] is to be believed - a technical sense that is distinct from that which I wish to entertain. However, it has also been invoked in relation to, for example, the rectification of Brownian motion into propulsive forces by bacterial flagellar systems (Peskin et al. 1993), and this is highly relevant to my present concerns.
} 
transformation and the self-catabolism needed to generate again resource for recombination and re-entry into metabolic cycles, so that the process does not - for a time, and ceteris paribus - end.

To this virtuous metabolic architecture we could add such additional causal factors as the fluidity of the cell and - not unrelatedly - the weakness of biomolecular forces and interactions. These too are key, and in part the cell seems complex because it is only in combination that the various causal factors enable, indeed constitute, life. Without fluidity, no flux; without flux, the damaging accumulation of matter; if strong forces, less fluidity, less flux, less thermally driven molecular state space exploration. With fluidity and weak interactions, on the other hand, comes the exploratory character of life. Living systems, from their lowest biophysical levels upwards and outwards, constantly probe their structural state space, committing no more than they must to a settled configuration of their parts. And in service of this, within their range of operating temperatures, they are avid exploiters of ambient thermal energy.

\section{The cell as Schrödinger Machine}

It would appear, then, that we have what amounts to a revised and updated answer to Schrödinger's question, in the $75^{\text {th }}$ anniversary year of the lectures he delivered and which formed the basis for 'What is Life?'. Aiming for a (moderately) concise encapsulation, we might say that the cell, the basic unit of life, is

a bounded, open, fluid molecular system that persists through the transduction of thermal energy and the existence of an underlying dis-equilibrium-driven metabolic architecture that maintains the open character of the system while retaining its identity.

In natural living systems the metabolic driver is ATP, but it could be some other molecular species, and the ATP/ADP dis-equilibrium is established chemiosmotically, but again could perhaps be established via some other route.

Now if we grant that the cell is such a thing, and admit that it is doing chemical work to realise functional ends, then it is not unreasonable to think of the cell as a kind of machine. This will hold all the more when artificially engineered cells and kindred living systems developed by synthetic biologists are used routinely to perform specific functional tasks, as it seems certain that they shall be (Jones 2004; Luisi 2006). And in that case, perhaps the perspicacity of Schrödinger's partial answers to the biological question of questions would be fittingly honoured if such machines were called Schrödinger Machines.

Some will no doubt object to such a linguistic move on the basis that organisms are in various respects quite unlike conventional man-made machines (Nicholson 2013), a point from which presumably few would demur. But in a sense the disjunction is precisely what motivates the move. Yes, the differences from man-made machines are profound, but organisms are work-performing, function-fulfilling, material objects nonetheless - as the graphic works of physician Fritz Kahn, Schrödinger's contemporary and fellow German 
emigré, serve to remind us (Debschitz \& Debschitz 2013). The new term is to a certain extent the complement of the term 'artificial life'. Whereas artificial life, in its soft[ware] form, draws a parallel between various bio-inspired algorithmic constructions and living material systems, we now accentuate the similarities between those living systems and potential (as yet hypothetical) man-made systems that operate according to the same biochemical principles. Indeed, there will in future arguably be more value in having a term for systems that work the same way (i.e. via a driven, thermal energy-transducing metabolism), irrespective of the functions they fulfil or how they came into being, than there is in having a term like artificial life that attempts to confer some of the glory of the living on crude in silico approximations of aspects of biological systems while effectively drawing a veil over what life is.

\section{References}

Bianconi, E., et al. (2013) An estimation of the number of cells in the human body. Annals of Human Biology 40(6): 463-471.

Brzezinski, P. (2017) The Development of Cryo-Electron Microscopy. (Scientific Background on the Nobel Prize in Chemistry 2017.) The Royal Swedish Academy of Sciences.

Callender, R. and Dyer, B.D. (2015) The Dynamical Nature of Enzymatic Catalysis. Accounts of Chemical Research 48: 407-413.

Cooper, M.M. and Klymkowsky, M.W. (2013) The Trouble with Chemical Energy: Why Understanding Bond Energies Requires an Interdisciplinary Systems Approach. CBE-Life Sciences Education 12: 306-312.

Dawidowicz, E.A. (1987) Dynamics of Membrane Lipid Metabolism and Turnover. Annual Review of Biochemistry 56: 43-61.

Debschitz, T. von and U. von (2013) Fritz Kahn. Taschen.

Di Rienzo, C., et al. (2014) Probing short-range protein Brownian motion in the cytoplasm of living cells. Nature Communications 5:5891. (DOI: 10.1038/ncomms6891)

Dobson, C.M. (2003) Protein folding and misfolding. Nature 426: 884-890.

Dreyfus, B.W. et al. (2014) Students' reasoning about "high-energy bonds" and ATP: A vision of interdisciplinary education. Physical Review Special Topics - Physics Education Research 10, 010115.

Fried, S.D. and Boxer, S.G. (2017) Electric Fields and Enzyme Catalysis. Annual Review of Biochemistry 86: 387-415.

Glowacki, D.R., Harvey, J.N. and Mulholland, A.J. (2012) Taking Ockham's Razor to enzyme dynamics. Nature Chemistry 4: 169-176.

Hay, S. and Scrutton, N.S. (2012) Good vibrations in enzyme-catalysed reactions. Nature Chemistry 4: 161-168.

Hinkson, I.V. and Elias, J.V. (2011) The dynamic state of protein turnover: It's about time. Trends in Cell Biology 21(5): 293-303.

Huang, S. et al. (2005) Cell Fates as High-Dimensional Attractor States of a Complex Gene Regulatory Network. Physical Review Letters 94(128701): 1-4. 
Jones, R.A.L. (2004) Soft Machines: Nanotechnology and Life. Oxford University Press.

Klinman, J.P. and Kohen, A. (2013) Hydrogen Tunneling Links Protein Dynamics to Enzyme Catalysis. Annual Review of Biochemistry 82: 471-496.

Klinman, J.P., et al. (2017) Origins of Enzyme Catalysis: Experimental Findings for C-H Activation, New Models, and Their Relevance to Prevailing Theoretical Constructs. Journal of the American Chemical Society 139(51): 18409-18427.

Kohen, A. (2014) Role of Dynamics in Enzyme Catalysis: Substantial versus Semantic Controversies. Accounts of Chemical Research 48: 466-473.

Koshland, D.E. (1995) The Key-Lock Theory and the Induced Fit Theory. Angewandte Chemie International Edition 33(23-24): 2375-2378.

Kühlbrandt, W. (2014) The Resolution Revolution. Science 343(6178): 1443-1444.

Linding, R., et al. (2003) Protein disorder prediction: implications for structural proteomics. Structure 11(11): 1453-1459.

Luisi, P.L. (2006) The Emergence of Life: From Chemical Origins to Synthetic Biology. Cambridge University Press.

McCammon, J.A. and Harvey, S.C. (1987) Dynamics of Proteins and Nucleic Acids. Cambridge University Press.

Méthot, P.-O. and Alizon, S. (2014) What is a pathogen? Towards and process view of host-parasite interactions. Virulence 58: 775-785.

Muñoz, V. and Cerminara, M. (2016) When fast is better: protein folding fundamentals and mechanisms from ultrafast approaches. Biochemical Journal 473: 2545-2559.

Murphy, M.P. (2009) How mitochondria produce reactive oxygen species. Biochemical Journal 417(Pt 1): $1-13$.

Natoli, G. (2010) Maintaining Cell Identity through Global Control of Genomic Organization. Immunity 33: $12-24$

Nicholls, D.G. (1982) Bioenergetics (1st Edition). Academic Press.

Nicholls, D.G. and Ferguson, S.J. (2013) Bioenergetics 4. Elsevier Ltd.

Nicholson, D.J. (2013) Organisms ₹ Machines. Studies in History and Philosophy of Biological and Biomedical Sciences 44(4 Pt B): 669-678.

O'Malley, M.A. and Dupré, J. (2009) Varieties of Living Things: Life at the Intersection of Lineage and Metabolism. Philosophy and Theory in Biology 1:e003. (DOI: 10.3998/ptb.6959004.0001.003)

Pauling, L. (1946) Molecular Architecture and Biological Reactions. Chemical and Engineering News 24(10): 1375-1377.

Peskin, C.S., et al. (1993) Cellular Motions and Thermal Fluctuations: The Brownian Ratchet. Biophysical Journal 65: 316-324.

Rassam, P. et al. (2015) Supramolecular assemblies underpin turnover of outer membrane proteins in bacteria. Nature 523(7560): 333-336.

Rich, P. (2003) The cost of living. Nature 421: 583.

Schrödinger, E. (1944) What is Life? (Canto edition, published together with Mind and Matter and Autobiographical Sketches, 1992.) Cambridge University Press. 
Scully, J.L. (2004) What is a disease? EMBO reports 5(7): 650-653.

Shapiro, J. (2011) Evolution: A View from the $21^{\text {st }}$ Century. FT Press Science.

Steer, M.W. (1988) Plasma Membrane Turnover in Plant Cells. Journal of Experimental Botany. 39(205): 987-996.

Storey, R.D. (1992) Textbook Errors \& Misconceptions in Biology: Cell Energetics. The American Biology Teacher 54(3): 161-166.

Törnroth-Horsefield, S. and Neutze, R. (2008) Opening and closing the metabolite gate. Proceedings of the National Academy of Sciences 105(50): 19565-19566.

Warshel, A. and Bora, R.P. (2016) Perspective: Defining and quantifying the role of dynamics in enzyme catalysis. The Journal of Chemical Physics 144(180901): 1-17.

Wright, P.E. and Dyson, H.J. (2015) Intrinsically Disordered Proteins in Cellular Signaling and Regulation. Nature Reviews Molecular Cell Biology 16(1): 18-29.

Zhao, Q. (2017) On the indirect relationship between protein dynamics and enzyme activity. Progress in Biophysics and Molecular Biology 125: 52-60.

Zhou, H.-X., et al. (2008) Macromolecular Crowding and Confinement: Biochemical, Biophysical, and Potential Physiological Consequences. Annual Review of Biophysics 37: 375-397. 